\title{
EDUCAÇÃO EM SAÚdE PARA A PREVENÇÃO DE DOENÇAS: UMA REVISÃO DA LITERATURA
}

\section{ARTIGO DE REVISÃO}

GITIRANA, José Valdeci Almeida1, FONSECA, Rosa Maria Batista Pinheiro da ${ }^{2}$, PILONETO, Fábio Marmentini ${ }^{3}$, BEVILAQUA, Luis Felipe Gaia ${ }^{4}$, ASSIS, Ingrid de ${ }^{5}$, CARDOSO, Ronald de Oliveira ${ }^{6}$

GITIRANA, José Valdeci Almeida. Et al. Educação em saúde para a prevenção de doenças: uma revisão da literatura. Revista Científica Multidisciplinar Núcleo do Conhecimento. Ano. 06, Ed. 11, Vol. 08, pp. 134-147. Novembro 2021. ISSN: 24480959, Link de acesso: https://www.nucleodoconhecimento.com.br/saude/educacaoem-saude, DOI: 10.32749/nucleodoconhecimento.com.br/saude/educacao-emsaude

\section{RESUMO}

Atualmente políticas públicas, ações governamentais e distintos programas envolvendo a Educação em Saúde (ES) são realizados em prol da saúde populacional. No que tange a temática da ES, esta é um tema multifacetado, convergente para diversas concepções pessoais, seja na área da educação ou saúde, podendo resultar em divergentes compreensões. Mediante à complexidade do tema, a problemática ocorre pela falta de compreensão do que realmente é a ES voltada para a população, dissolvendo a importância da ES para medidas preventivas contra distintas doenças, o que gera alto custo orçamentário na saúde pública. Tendo em vista este contexto, o presente artigo, tem como questão

\footnotetext{
${ }^{1}$ Graduado em Farmácia, pós-graduado em Análises Clínicas, Atualmente acadêmico de medicina.

${ }^{2}$ Acadêmico de Medicina.

${ }^{3}$ Graduado em administração. Graduado em comércio exterior, atualmente acadêmico de medicina.

${ }^{4}$ Graduado em engenharia de alimentos, pós-graduado em engenharia de processos industriais, CBA em gestão empresarial, e atualmente acadêmico de Medicina.

${ }^{5}$ Acadêmico de Medicina.

${ }^{6}$ Acadêmico de Medicina.
}

RC: 101474

Link de acesso: https://www.nucleodoconhecimento.com.br/saude/educacao-emsaude 
norteadora: Como a educação em saúde pode contribuir para a prevenção de doenças na população? O estudo teve como objetivo geral apresentar a ES para a Prevenção de Doenças (PD), ramificando em abordar os seus conceitos, bem como destacar as bases legais que asseguram a ES à população. Para tanto, o estudo foi realizado através de uma revisão da literatura pelo site de busca "Google Acadêmico", "Scielo" e "PubMed". Por meio dos descritores: Educação em Saúde; Educação em Saúde como prevenção de doenças; Educação em Saúde para a população, onde foram escolhidos estudos que apresentaram a ES voltada para a PD. Conclui-se que a ES é a educação para o autoconhecimento e reflexão sobre sua própria saúde, bem como a plena consciência de que algo vai mal, atribuindo a reflexão crítica do sujeito quanto a seus hábitos e medidas preventivas. Ela é aplicada por meio do currículo escolar desde o ensino básico como também por programas governamentais nos núcleos de saúde, de modo a educar a sociedade para a conscientização sobre medidas preventivas e a promoção de uma melhor qualidade de vida, prevenindo, assim, a propagação de doenças.

Palavras-chave: Educação em Saúde, Prevenção de doenças, Promoção da Saúde, População.

\section{INTRODUÇÃO}

A Educação em Saúde (ES) é uma prática social cujo processo de aprendizagem contribui para a formação da consciência crítica pessoal no que se refere os próprios problemas de saúde, fazendo com que o cidadão reflita sobre sua realidade, e a partir dela, busque soluções e a organização em prol de sua saúde e saúde coletiva, de modo a resultar em uma Promoção da Saúde (PS) (BISCARDE; PEREIRASANTOS; SILVA, 2014).

As origens e concepções da PS iniciou-se com o advento da ES no início do século XX, por meio da observação do decréscimo dos índices de adoecimentos decorrentes de práticas educativas realizadas por "higienistas[7]" do período (ENRIA; STAFOLANI, 2010). Naquela época, o significado da PS atribuía-se às 
ações da ES, com o intuito de uma melhor qualidade de vida. Contudo, a ES na atualidade apresenta uma característica amplificada, uma vez que é considerada um dos principais dispositivos para a viabilização da PS, balizando para o desenvolvimento da responsabilidade individual e a Prevenção de Doenças (PD) (LOPES; SARAIVA; XIMENES, 2010; FALKENBERG et al., 2014; SPERANDIO; SPERANDIO, 2018).

Apesar da ES ser entendida como uma das medidas para a PD (JANINI; BESSLER; VARGAS, 2015; SPERANDIO; SPERANDIO, 2018), ela é um tema multifacetado, convergente para diversas concepções pessoais, seja para pessoas da área da educação, ou da área da saúde, podendo resultar em divergentes compreensões. (SCHALL; STRUCHINER, 1999).

Mediante à complexidade do tema, a problemática ocorre pela falta de compreensão do que realmente é a ES voltada para a população, dissolvendo a importância da ES para medidas preventivas contra distintas doenças. Devido a isso, muitas doenças que poderiam ser evitadas são frequentemente evidenciadas causando um grande impacto no orçamento público. Portanto, o presente estudo primou em responder à pergunta: Como a educação em saúde pode contribuir para a prevenção de doenças na população? Por meio de uma revisão da literatura, o objetivo geral buscou apresentar como a ES pode promover a PD, ramificando em abordar os seus conceitos, bem como destacar as bases legais que asseguram a ES à população.

Para tanto, o estudo foi realizado através de uma revisão da literatura pelo site de busca "Google Acadêmico", "Scielo" e "PubMed". Por meio dos descritores: Educação em Saúde; Educação em Saúde como prevenção de doenças; Educação em Saúde para a população. Como modo de inclusão foram escolhidos os artigos e documentos entre os anos de 2018 a 2020, compondo a estrutura necessária para o alcance do objetivo desejado. Posteriormente, 20 artigos foram encontrados, destes, 8 foram selecionados. Foram inclusos os artigos que respondessem ao objetivo e questão norteadora e estivessem dentro do recorte temporal proposto. Foram 
excluídos os estudos voltados somente para profissionais da saúde ou graduandos da área da saúde.

O tema é relevante nos dias atuais uma vez que a ES pode influenciar na capacidade de reflexão da população para o combate a distintas doenças, resultando em melhores hábitos de vida. Pode ser relevante para pessoas leigas ou para estudantes e profissionais do campo acadêmico, servindo como fonte para futuros estudos pois é esclarecedor do conceito da ES para a população como indissociável da PD brindando uma linguagem de fácil compreensão.

\section{A INDISSOCIABILIDADE DA EDUCAÇÃO EM SAÚDE E PREVENÇÃO DE DOENÇAS}

A ES é planificada e aplicada de distintas maneiras, tal modalidade de ensino pode ser voltada para graduandos da área da saúde; para profissionais da saúde; para a formação escolar e para a população de modo geral. Com efeito, tende a ser compreendida de distintas maneiras, indo de encontro da formação cultural e social de cada um. No entanto, o presente estudo enfatiza a ES para a população como indissociável da PD, uma vez que a ES se "[...]liga aos objetivos gerais da saúde pública: prevenção de doenças, proteção e promoção da saúde do indivíduo, da família e da comunidade". (PIMONT, 1997, p. 17).

Para contextualizar melhor sobre a compreensão dessa indissociabilidade, é importante destacar que a ES é um:

Processo educativo de construção de conhecimentos em saúde que visa à apropriação temática pela população [...]. Conjunto de práticas do setor que contribui para aumentar a autonomia das pessoas no seu cuidado e no debate com os profissionais [...] a fim de alcançar uma atenção de saúde de acordo com suas necessidades. (BRASIL, 2012, p. 19).

Conforme apresentado no "Glossário Temático: gestão do trabalho e da educação em saúde" a ES ocasiona a autonomia desejável para que as pessoas possam se expressar eficazmente de modo a evidenciar suas necessidades específicas em

RC: 101474

Link de acesso: https://www.nucleodoconhecimento.com.br/saude/educacao-emsaude 
seus atendimentos clínicos, contribuindo para o atendimento do profissional da saúde a tratar prontamente as enfermidades, bem como prevenir outras doenças secundárias. Complementando essa definição, é apresentado no documento da Fundação Nacional de Saúde (FUNASA) Brasil (2007), que a ES é uma prática social, reafirmando que sua intenção tece em uma transformação para qualquer tipo de pessoa "[...] rompendo com o paradigma da concepção estática de educação como transferência de conhecimentos, habilidades e destrezas". (BRASIL, 2007, p. 20).

Pimont (1997, p. 15) resume e analisa diferentes conceitos adotados, enfatizando que a ES, objetiva em proporcionar:

1) A consciência do direito à saúde, dentro do grau de desenvolvimento do grupo social no qual vive o indivíduo; 2) O conhecimento sobre saúde; 3) O conhecimento dos serviços para a saúde que a comunidade possua e sua consequente utilização; 4) O conhecimento e a utilização das práticas de saúde.

No entanto, o autor assimila que para esse ensino ser transformado em conhecimento de modo a promover a PS e a PD, é necessário que um planejamento seja estruturado, atenuando uma adequada metodologia educativa, composta por instrumentos auxiliares, podendo então resultar em um constante processo educativo.

Segundo Altuve (1996), para que a ES se transforme em conhecimento, a organização da comunidade já se torna um método que produz a mudança social, pois em meio aos esforços e técnicas, sua aplicação resulta na indução e promoção da mudança social significante, englobando todos os setores sociais, como a relação dos níveis econômicos, políticos e educativos.

Portanto, de acordo com os autores citados, a ES pode ser indissociável da PD para a população, quando transmitida metodologicamente. Porém o presente estudo não pretende aprofundar nessas metodologias, outrossim, conceituar a ES como PD para a população.

RC: 101474

Link de acesso: https://www.nucleodoconhecimento.com.br/saude/educacao-emsaude 


\subsection{BASES LEGAIS PARA A EDUCAÇÃo EM SAÚdE PARA A POPULAÇÃO}

Com o foco na ES no Brasil, em agosto de 1971 foi criada a Lei 5692, reformulando o currículo escolar no ensino primário e médio implementando o ensino voltado para área de saúde conforme apresentado no artigo 7:

Art. 7ํ Será obrigatória a inclusão de Educação Moral e Cívica, Educação Física, Educação Artística e Programas de Saúde nos currículos plenos dos estabelecimentos de $I^{\circ}$ e $2^{\circ}$ graus, observado quanto à primeira o disposto no Decreto-Lei n. 369, de 12 de setembro de 1969. (Vide Decreto no 69.450, de 1971)

Posteriormente programas como "Saúde na escola", foram utilizados como estratégia intersetorial desenvolvidos no espaço escolar e em seu entorno, articulando ações voltadas para a PS e da qualidade de vida, juntamente da reflexão sobre a PD e conhecimento do Sistema Único de Saúde (SUS) (BRASIL, 2012). Esse contexto pode ser pontuado pelo fato das ações para a ES serem implementadas ao longo da vida, desde a primeira fase da formação escolar, para que as pessoas desenvolvam um senso crítico, reflexivo e consciente gradualmente.

Irrompendo positivamente 0 alcance de programas voltados para a ES e PD, distintas leis foram criadas, conforme as bases legais apresentadas no quadro 1 abaixo:

RC: 101474

Link de acesso: https://www.nucleodoconhecimento.com.br/saude/educacao-emsaude 


\title{
Quadro 1 - Bases legais da Educação em Saúde.
}

\begin{abstract}
A Lei n. ${ }^{\circ}$ 8.080/1990, que regulamenta o SUS, em seu Capítulo I, Art. $5^{\circ}$, Item III, define como um dos objetivos e atribuições deste Sistema: "a assistência às pessoas por intermédio de ações de promoção, proteção e recuperação da saúde, com a realização integrada das ações assistenciais e das atividades preventivas". A mesma Lei, em seu Capítulo II, Artigo 7_ ${ }^{\circ}$, Item VIII, estabelece a
\end{abstract} "participação da comunidade."

A Lei $\mathbf{N}^{\circ} \mathbf{8 . 1 4 2}$, de 28 de dezembro de 1990, define a participação da comunidade na gestão SUS e no seu artigo $1^{\circ}$ "as instâncias colegiadas de controle social: I) a Conferência de Saúde, e II) o Conselho de Saúde."

A Norma Operacional Básica - SUS 1/1996 (NOB/96), que redefine as responsabilidades dos estados, do Distrito Federal e da União e consolida as responsabilidades dos municípios, cita como um dos papéis do gestor federal e do estadual a "Educação em Saúde

A Educação em Saúde na Funasa está ancorada em seu Regimento Interno, Portaria GM n ${ }^{\circ} 1.776$ de 8/9/2003, assinada pelo Ministro da Saúde que estabelece as competências da Assessoria de Comunicação e Educação em Saúde-Ascom e seus setores, dentro das competências dos demais setores do órgão. (...)

Lei n. 9.394, de 20 de dezembro de 1996 (1996). Estabelece as diretrizes e bases da educação nacional.

DCN - EF 1988 - Estabelecer as Diretrizes Curriculares Nacionais para o Ensino Fundamental: Saúde articulada à educação e à vida cidadã.

PCN - CN 1988 - Estabelecer as Referências Nacionais Comuns para o Ensino das Ciências Naturais: Saúde como estado de equilíbrio dinâmico do corpo.

PCN - TT Saúde 1988 - Apresentar a saúde como um Tema Transversal: Educação para a saúde

PCN - EM 1999 - Apresentar os Parâmetros Curriculares Nacionais para o Ensino Médio: Degradação ambiental e agravos à saúde humana.

PCN + 2002 - Apresentar Orientações Curriculares complementares aos PCN: Saúde e as condições de vida das pessoas.

OCEM 2006 - Apresentar sugestões para as práticas pedagógicas e organização dos

currículos do Ensino Médio: Ambiente e saúde.

DCNEB 2013 - Estabelecer Orientações Nacionais para a Educação Básica. Saúde como campo de conhecimento e de serviços.

BNCC - $\mathbf{1}^{2}$ versão 2015 - Estabelecer uma Base Nacional Comum para os currículos da Educação Básica, com versão inicial disponibilizada para consulta pública, estabelecendo cuidado com a saúde da população.

BNCC - 2 ${ }^{\mathrm{a}}$ versão 2016 - Estabelecer uma Base Nacional Comum para os currículos da Educação Básica, com versão elaborada após contribuições da sociedade, para o ambiente e saúde.

BNCC $3^{a}$ versão 2016 - Estabelecer uma Base Nacional Comum para os currículos da Educação Básica com versão disponibilizada após contribuições institucionais, para promoção dos cuidados com a saúde.

BNCC versão final (EI e EF) 2016 - Estabelecer uma Base Nacional Comum para os currículos da Educação Infantil e do Ensino Fundamental, compondo a versão final, com o objetivo de promover os cuidados com a saúde.

BNCC EM 2018 - Estabelecer uma Base Nacional Comum para os currículos do Ensino Médio, promoção do cuidado com a saúde.

Fonte: Adaptado de Brasil (2012); Sousa, Guimarães e Amantes (2019).

RC: 101474

Link de acesso: https://www.nucleodoconhecimento.com.br/saude/educacao-emsaude 
O quadro apresenta as leis, onde é destacada algumas bases educacionais para a aplicabilidade da ES na escola, mesmo que tais diretrizes são voltadas ao âmbito escolar, os propósitos curriculares balizam na formação de pessoas que compreendam a importância da ES e que através desse conhecimento possam promover o cuidado com a saúde pessoal e populacional, compondo também a benfeitoria da PD.

$\mathrm{Na}$ atualidade as Nações Unidas apresentam o terceiro objetivo na "Agenda 2030", e com o apoio mundial, pretende-se por meio da ação, "Assegurar uma vida saudável e promover o bem-estar para todas e todos, em todas as idades" (ODS, 2021) independentemente da classe social, raça ou cor.

O lugar na agenda de 2030 vai de encontro com a perspectiva de SHALL (1999), quando atenua que a definição para a ES precisa ser mais ampla do que simplesmente uma ação resultante da PS, uma vez que ela voltada para a população perpassa um processo abrangente da participação ativa de toda a população no contexto de sua vida cotidiana e não apenas das pessoas que possuem risco de adoecer, sendo então uma maneira de prevenir doenças às pessoas saudáveis, instaurando uma melhor qualidade de vida e maior bem-estar a todos.

\subsection{ESTUDOS RELACIONADOS À PREVENÇÃO DE DOENÇAS POR MEIO DA EDUCAÇÃO EM SAÚDE}

$\mathrm{Na}$ sequência, serão apresentados os principais achados acerca da PD como resultado da aplicação da ES, disponíveis na literatura científica, entre os anos de 2018 a 2020. A seguir, no quadro 2 é feita uma síntese dos 8 artigos previamente selecionados apresentados em ordem cronológica, onde posteriormente serão explanadas as metodologias utilizadas em cada estudo.

RC: 101474

Link de acesso: https://www.nucleodoconhecimento.com.br/saude/educacao-emsaude 
Quadro 2 - Estudos relacionados à Educação em Saúde como prevenção de doenças.

\begin{tabular}{|c|c|}
\hline Autor & Objetivo \\
\hline $\begin{array}{l}\text { Dias et al. } \\
(2018)\end{array}$ & $\begin{array}{l}\text { Relatar a experiência de uma } \\
\text { ação de educação em saúde } \\
\text { com escolares do ensino } \\
\text { fundamental de uma escola } \\
\text { pública em zona rural. }\end{array}$ \\
\hline $\begin{array}{l}\text { Salci, } \\
\text { Meirelles e } \\
\text { Silva (2018) }\end{array}$ & $\begin{array}{l}\text { Compreender como os } \\
\text { integrantes da Estratégia Saúde } \\
\text { da Família se auto-organizam } \\
\text { para a educação em saúde, no } \\
\text { manejo e prevenção das } \\
\text { complicações crônicas do } \\
\text { diabetes mellitus na Atenção } \\
\text { Primária à Saúde }\end{array}$ \\
\hline
\end{tabular}

Conclusão

Gomes et Relatar ação educativa em al. (2019) grupo de homens visando buscar melhor compreensão trabalhado entre os homens. sobre o câncer de pênis e as medidas de prevenção e detecção precoce.

Figueiredo; Levar à criação de políticas As Políticas Públicas são Antunes e públicas de educação em imprescindíveis para a De Miranda saúde, com atendimento comunicação e o fortalecimento (2019) escolar específico e devido de todas as medidas e diretrizes encaminhamento dos alunos das áreas de educação e saúde. A 
portadores de obesidade às interdisciplinaridade promoverá as unidades de saúde transformações desejadas, por meio de ações prevencionistas, com 0 intuito de reduzir 0 acometimento da obesidade e suas consequências, nas faixas etárias mais jovens, como também, a redução de doenças cardiovasculares na fase precoce da vida e na fase adulta.

Ricci et al. Relatar a experiência de Realização da educação em (2019) acadêmicas da Universidade Federal de Mato Grosso do Sul (UFMS) durante ação saúde sobre as Infecção Sexualmente Transmissível (IST) desenvolvida com o grupo de se proporcionar o diálogo, gestantes de uma Unidade de interação, a troca de experiências, Básica de Saúde em Campo saberes e vivências, e a Grande - MS (UBS). construção conjunta do conhecimento, sendo então, tais elementos Evidenciados como importantes para a promoção da saúde e a prevenção de doenças, e para o desenvolvimento de uma consciência crítica e reflexiva das gestantes acerca de seu processo saúde-doença, ao torná-las ativas no processo de aprendizagem.

De Sousa Conhecer o trabalho de Percebe-se que é imprescindível o Gonçalves educação em saúde de uma constante aprimoramento do et al. (2020) unidade básica de saúde do conhecimento e prática dos 
município de Imperatriz - MA.

Lima et al. Descrever a experiência como (2020)

Costa et al. Relatar uma ação de educação (2020) acadêmicas de enfermagem nas atividades de educação em saúde para a prevenção de doenças cardiovasculares em idosos. profissionais para desenvolver as ações de educação em saúde. É necessário a expansão das ações educativas dentro da área de responsabilidade de cada equipe, assim como uma participação mais ativa de todos os membros da equipe de estratégia da família, bem como os profissionais do núcleo de apoio à saúde da família (NASF).

As práticas de educação em saúde são transformadoras e trazem contribuições importantes para enfermagem gerontológica, pois possibilita o exercício do pensamento crítico-reflexivo e contribui para a formação de profissionais comprometidos para atuarem em um modelo assistencial, pautado nas evidências cientificas e capacitados a atender as necessidades da população idosa, que vem crescendo com o passar dos anos.

A ação desenvolvida serviu como em saúde ocorrida no âmbito da mecanismo consolidador dos atenção primária de saúde, cuja aspectos que constituem a proposta perpassava pela educação em saúde, reiterando a 


\begin{tabular}{l|lll} 
prevenção do câncer de mama & função fundamental & da \\
a partir do reconhecimento & enfermagem no processo do & nón \\
prévio de suas manifestações, & cuidar. & \\
bem como da manutenção de & & \\
hábitos saudáveis, visando a & \\
promoção da saúde dessas & \\
mulheres.
\end{tabular}

Fonte: $O$ autor.

Dias et al. (2018), através de um relato de experiência com método de atividade extraclasse do componente curricular "Projeto Integrador Científico da Graduação em Enfermagem" da Faculdade Verde Norte, realizaram em outubro de 2016 na Escola Municipal Odilon Antunes, no Povoado de Poções da cidade de Monte Azul, uma ação de educação em saúde com o envolvimento de 30 alunos, 03 professores e 09 pais. A ação ocorreu nas etapas de exposição dialógica do conteúdo e na exibição de vídeo disparador de discussão, sendo debatidas em roda de conversas com público. Desse modo, o estudo proporcionou medidas preventivas para a contaminação por doenças parasitárias.

Salci; Meirelles e Silva (2018) realizaram um estudo qualitativo, compondo o Pensamento Complexo como referencial teórico e a pesquisa avaliativa como referencial metodológico. Para a coleta de dados, 38 profissionais de saúde entrevistaram os pacientes com Diabetes Mellitus. Os agentes de saúde observaram e analisaram os prontuários médicos dos pacientes, por meio da triangulação dos dados com o apoio do software ATLAS.ti. Os autores observaram que de acordo com a complexidade do tema multifacetado, a ES pode promover a PD primárias e secundárias.

Corroborando tal complexidade, Figueiredo; Antunes e De Miranda (2019), realizaram uma pesquisa de revisão bibliográfica, aplicando o método de pesquisa exploratória. Os autores relacionaram a prevenção de comorbidades e doenças 
cardiovasculares às políticas públicas de ES no âmbito escolar. Em conseguinte, destacaram que a interdisciplinaridade que envolve a ES pode promover as transformações desejadas nos alunos, por meio de ações prevencionistas, prevenindo doenças cardiovasculares na fase da infância e fase adulta.

Lima et al. (2020) em um estudo semelhante, porém voltado ao público de idosos, observaram por meio de um relato de experiência, através do método descritivo, com abordagem qualitativa, vivenciado no "Programa de Atenção à Saúde do Idoso - PROASI", que as atividades de ES servem como ferramenta na prevenção de doenças cardiovasculares, ocasionando um pensamento crítico-reflexivo à própria saúde e qualidade de vida.

Já Gomes et al. (2019) por meio do estudo de cunho descritivo exploratório do tipo relato de experiência, aplicaram uma atividade realizada no mês de março de 2018 em uma igreja localizada na periferia de Belém/Pará. O público participante foi composto de vinte e três homens na faixa etária de 18 a 50 anos que foram submetidos às palestras com a apresentação de banners; explicação do câncer peniano; os cuidados que se deve ter para a PD e demonstração de próteses penianas. Os autores observaram por meio da roda de conversa com os participantes, que poucos conheciam sobre a temática do câncer peniano. Portanto, os autores alertaram sobre a importância da implementação de programas da ES voltada para a PD como o câncer peniano, pois com programas voltados para a ES essa e outras doenças podem ser consideravelmente diminuídas na população.

Ainda no contexto da prevenção do câncer, Costa et al. (2020), realizaram um estudo descritivo tendo uma abordagem qualitativa, do tipo relato de experiência. $O$ estudo foi realizado no âmbito hospitalar, encontrado na região periférica da cidade de Belém- PA, em uma unidade de saúde com profissionais que promovem uma modalidade de tratamento de saúde voltada para o nível primário das doenças. Os tratamentos voltados ao público feminino, se consolidam na implantação do "Programa Saúde da Mulher", no qual as pacientes são munidas de atendimentos com o acompanhamento do pré-natal; parto; pós-parto; atendimento para famílias; 
programas para a prevenção do câncer de mama e uterino; IST. Esse programa é composto por profissionais de diversificadas áreas da saúde, juntamente dos variados tipos de análises clínicas para a adoção de medidas preventivas, fomentando o diagnóstico prévio de patologias como o câncer. Com a implementação de uma ação educativa no programa, profissionais da saúde acompanharam as pacientes em suas consultas, e similarmente ao estudo de Gomes et al. (2019) expuseram folders; apresentações anatômicas da mama; entre outras atividades educativas e interativas. Logo, os autores elucidaram que as 16 participantes tiveram uma maior reflexão sobre a importância do autocuidado e compreenderam a importância da ES para a prevenção do câncer de mama.

No que tange a essa reflexão citada por Gomes et al. (2019), Ricci et al. (2019) corroboram que tal processo de consciência reflexiva ocorre mediante o diálogo; interação; troca de experiências; saberes e vivências e a construção conjunta do conhecimento, sendo então, tais elementos evidenciados como importantes para a PS e a PD. Essa observação foi evidenciada através do estudo ocorrido em 15 de março de 2017, no período vespertino na sala de reuniões de uma Unidade Básica de Saúde de Campo Grande - MS. Foi utilizado uma metodologia de modo estratégico composto por práticas de ludicidade com o intuito de interatividade dos participantes para que por meio da prática pedagógica eles pudessem adquirir o entendimento do tema em questão. Inicialmente as participantes gestantes foram convidadas por meio de convocações pelos agentes comunitários na região da unidade de saúde. Posteriormente $A$ ação se iniciou com a técnica quebra gelo "Passando o bebê", na qual as participantes eram apresentadas se identificando com o seu nome, idade gestacional e escolha do nome do filho (a). Por meio das dinâmicas, as gestantes se conscientizaram sobre as doenças que poderiam ser prevenidas durante a gestação bem como a PD no próprio bebê.

Já para que essa consciência reflexiva envolva a família, De Sousa Gonçalves et al. (2020), realizaram um trabalho de natureza quantitativa com abordagem analítica, a partir de uma coleta retrospectiva das ações de ES realizadas no período de fevereiro a setembro de 2019. A pesquisa foi realizada em uma Unidade Básica de 
Saúde (UBS) do município de Imperatriz no estado do Maranhão, na qual foi composta por 3 equipes de Estratégia da Saúde da Família. Os dados foram coletados a partir do acervo documental com registros das ações de ES realizadas na UBS e nas escolas da área, coberta por cada equipe no período supracitado, disponibilizados pela direção da unidade de saúde. Os autores analisaram que por meio da ES não somente as doenças crônicas, sexuais ou infecciosas podem ser prevenidas, mas também medidas profiláticas podem ser resultantes de programas que auxiliam essas famílias.

\section{CONSIDERAÇÕES FINAIS}

A ES pode promover a PD por meio de programas de políticas públicas voltadas para o implemento de programas educativos da ES nas escolas, UBS, redes hospitalares bem como em postos de saúde. Para tanto, seus conceitos podem ser amplamente apresentados, uma vez que a ES é um tema complexo e multifacetado.

Através dos achados, considera-se que a ES pode estimular a PD juntamente da PS para a população, quando houver engajamento desse público-alvo através das atividades metodologicamente planejadas e aplicadas com o aporte de instrumentos específicos para o desenvolvimento do aprendizado. No que tange à PD são diversas as profilaxias encontradas nos estudos, sendo elas: cardiovasculares, diabetes, doenças crônicas não transmissíveis, doenças infecciosas sexualmente transmissíveis, doenças parasitárias, canceres, entre outras existentes.

No contexto das leis e bases legais para a ES, visa-se uma grande expectativa nos estudantes das fases iniciais e ensino médio, uma vez que o processo educativo não é imposto na fase adulta e sim pode ser construído durante o decorrer da vida, tornando-se conhecimento.

Portanto, em resposta a questão norteadora, conclui-se que a ES é a educação para - autoconhecimento e reflexão sobre sua própria saúde, bem como a plena consciência de que algo vai mal, atribuindo a reflexão crítica do sujeito quanto a 
seus hábitos e medidas preventivas, prevenindo, assim, doenças. Ela é aplicada por meio do currículo escolar desde o ensino básico como também por programas governamentais nos núcleos de saúde, de modo a educar a sociedade para a conscientização sobre medidas preventivas e a promoção de uma melhor qualidade de vida.

\section{REFERÊNCIAS}

ABREU JUNIOR, Laerthe de Moraes; CARVALHO, Eliane Vianey de. O discurso médico-higienista no Brasil do início do século XX. Trabalho, Educação e Saúde, v. 10, p. 427-451, 2012.

ALTUVE. R. L. O. La organización de la comunidad como un proceso educativo. Cuadernos de la Escuela de Salud Pública (Caracas) 4:9-28, 1966

BISCARDE, Daniela Gomes dos Santos; PEREIRA-SANTOS, Marcos; SILVA, Lília Bittencourt. Formação em saúde, extensão universitária e Sistema Único de Saúde (SUS): conexões necessárias entre conhecimento e intervenção centradas na realidade e repercussões no processo formativo. Interface-Comunicação, Saúde, Educação, v. 18, p. 177-186, 2014.

BRASIL. Fundação Nacional de Saúde. Diretrizes de educação em saúde visando à promoção da saúde: documento base - documento I/Fundação Nacional de Saúde -Brasília: Funasa, 2007.

. Ministério da Saúde (MS) Glossário temático: gestão do trabalho e da educação na saúde. Brasília DF, 2012. Disponível em: https://bvsms.saude.gov.br/bvs/publicacoes/glossario_gestao_trabalho_2ed.pdf Acesso em: 03/07/2021. . Lei; LEI, №. 5.692, DE 11 DE AGOSTO DE 1971. Fixa Diretrizes e Bases para o ensino de, v. 1, p. 6377, 1971. 
. Lei n. 9.394, de 20 de dezembro de 1996 (1996). Estabelece as diretrizes e bases da educação nacional. Brasília, DF: Ministério da Educação. Disponível em: http://portal.mec.gov.br/seed/arquivos/pdf/tvescola/leis/ lein9394.pdf Acesso em 20 de ago. 2021.

COSTA, Paula Valéria Dias Pena et al. A educação em saúde como ferramenta no combate ao câncer de mama: relato de experiência. Research, Society and Development, v. 9, n. 10, p. e6389108912-e6389108912, 2020.

DE SOUSA GONÇALVES, Romário et al. Educação em saúde como estratégia de prevenção e promoção da saúde de uma unidade básica de saúde. Brazilian Journal of Health Review, v. 3, n. 3, p. 5811-5817, 2020.

DIAS, Ernandes Gonçalves et al. Promoção de saúde na perspectiva da prevenção de doenças parasitárias por meio da educação em saúde com escolares do ensino fundamental. Revista de Epidemiologia e Controle de Infecção, v. 8, n. 3, p. 283285, 2018.

ENRIA, G.; STAFFOLANI, C. Contradicciones de los discursos que dificultan la transformación de las prácticas de promoción a la salud. Hacia la promoción de la salud, Calle, v. 15, n. 1, 2010.

FALKENBERG, Mirian Benites et al. Educação em saúde e educação na saúde: conceitos e implicações para a saúde coletiva. Ciência \& Saúde Coletiva, v. 19, p. 847-852, 2014.

FIGUEIREDO, Elisabeth Almeida; ANTUNES, Daniella Cavalcante; DE MIRANDA, Maria Geralda. Políticas públicas de educação em saúde para a prevenção de comorbidades e doenças cardiovasculares. Revista da Seção Judiciária do Rio de Janeiro, [S.I.], v. 23, n. 45, p. 141-160, jul. 2019. ISSN 2177-8337.

RC: 101474

Link de acesso: https://www.nucleodoconhecimento.com.br/saude/educacao-emsaude 
GOMES, Ana Cláudia Ferreira et al. Educação em saúde para prevenção do câncer de pênis: relato de experiência. Brazilian Journal of Health Review, v. 2, n. 4, p. 2961-2964, 2019.

JANINI, Janaina Pinto; BESSLER, Danielle; VARGAS, Alessandra Barreto de. Educação em saúde e promoção da saúde: impacto na qualidade de vida do idoso. Saúde em Debate, v. 39, p. 480-490, 2015. LOPES, M. S. V.; SARAIVA, K. R. O.;

LIMA, D. C.; GARCIA, M. P. .; LIMA, E. S. de .; BEZERRA, C. C. . Health education as a tool for the prevention of cardiovascular diseases in the Elderly Health Care Program. Research, Society and Development, [S. I.], v. 9, n. 10, p. e079107382, 2020. DOI: 10.33448/rsd-v9i10.7382. Disponível em: https://rsdjournal.org/index.php/rsd/article/view/7382. Acesso em: 28 aug. 2021.

OBJETIVOS DA ONU - ODS. Objetivo 3. Assegurar uma vida saudável e promover o bem-estar para todas e todos, em todas as idades. Disponível em: <https://gtagenda2030.org.br/ods/ods3/>. Acesso em: 25 ago. 2021

PIMONT, Rosa Pavone. A educação em saúde: conceitos, definições e objetivos. Boletín de la Oficina Sanitaria Panamericana (OSP); 82 (1), ene. 1977, 1977.

RICCI, Ana Patrícia et al. Infecções sexualmente transmissíveis na gestação: educação em saúde como estratégia de prevenção na atenção básica. Brazilian Journal of Health Review, v. 2, n. 1, p. 565-570, 2019.

SALCI, Maria Aparecida; MEIRELLES, Betina Hörner Schlindwein; SILVA, Denise Maria Guerreiro Vieira da. Educação em saúde para prevenção das complicações crônicas do diabetes mellitus na atenção primáriaa. Escola Anna Nery, v. 22, 2018.

SCHALL, Virgínia T.; STRUCHINER, Miriam. Educação em saúde: novas perspectivas. 1999.

RC: 101474

Link de acesso: https://www.nucleodoconhecimento.com.br/saude/educacao-emsaude 
SOUSA, Marta Caires; GUIMARÃES, Ana Paula Miranda; AMANTES, Amanda. A saúde nos documentos curriculares oficiais para o ensino de ciências: da lei de diretrizes e bases da educação à base nacional comum curricular. Revista Brasileira de Pesquisa em Educação em Ciências, p. 129-153, 2019.

SPERANDIO, Naiara; SPERANDIO, Natália Elvira. A educação em saúde enquanto prática transformadora da realidade social. Educação e Saúde: fundamentos e desafios, v. 1, n. 2, p. 7-14, 2018.

XIMENES, L. B. Análise do conceito de promoção da saúde. Texto Contexto Enferm., Florianópolis, v. 19, n. 3, jul./set. 2010, p. 461-468.

\section{APÊNDICE - REFERÊNCIA NOTA DE RODAPÉ}

7. Pessoas que faziam parte da doutrina do higienismo, uma doutrina nascida na metade do século XIX, quando os governantes deram maior atenção à saúde, juntamente da moral da população. Nessa época a doença foi considerada um fenômeno social envolvedor de todos os aspectos da vida humana, é por meio desse contexto que surge o tratamento da água, esgotos, iluminação nas ruas, a fim de controlar ou eliminar as pandemias (ABREU JUNIOR; CARVALHO, 2012).

Enviado: Setembro, 2021.

Aprovado: Novembro, 2021. 